\title{
Vision Spreadsheet: An Environment for Computer Vision
}

\author{
Scott Determan ${ }^{\ddagger *}$
}

\begin{abstract}
Vision Spreadsheet is an environment for computer vision. It combines a spreadsheet with computer vision and scientific python. The cells in the spreadsheet are images, computations on images, measurements, and plots. There are many built in image processing and machine learning algorithms and it extensible by writing python functions and importing them into the spreadsheet.
\end{abstract}

Index Terms—computer vision, spreadsheet, OpenCV

\section{Introduction}

Vision Spreadsheet is an application designed to explore and solve computer vision problems. It provides a visual environment and a familiar computational tool set to enable creative prototyping of computer vision algorithms. A novel interface using a spreadsheet of images encourages interactive and exploratory algorithm design. Computational scientists can leverage their existing knowledge of python, NumPy, SciPy, OpenCV, VIGRA, and other familiar technologies. Vision Spreadsheet aims to make the techniques of computer vision accessible to a wider audience.

Vision Spreadsheet is modeled after familiar numerical spreadsheets, such as MS Excel and Apple Numbers. In a numerical spreadsheet, each cell contains a number, with cells relating to each other by numerical expressions. In Vision Spreadsheet, each cell contains an image, with cells relating to each other by computer vision operations. As in a traditional spreadsheet, changes propagate automatically through the cells. Complex vision algorithms can be built-up cell-by-cell, interactively, with continuous visual feedback into the intermediate steps.

The cells within Vision Spreadsheet relate to each other through expressions that operate on images. For example, if the image in cell a 2 is the dilation of the image in cell a1, this is expressed as "dilate(a1)". The power of these expressions comes from the large library of functions available, including all of the image processing and machine learning algorithms from OpenCV. Furthermore, users can easily add their own functions using python, NumPy, and SciPy.

Vision Spreadsheet provides many tools to make it easier to explore the solution space of a vision problem.

- $\quad$ source images can be loaded, reload or looped through

* Corresponding author: scott.determan@gmail.com

+ Vision Spreadsheet

Copyright $\left({ }_{0} 2011\right.$ Scott Determan. This is an open-access article distributed under the terms of the Creative Commons Attribution License, which permits unrestricted use, distribution, and reproduction in any medium, provided the original author and source are credited.
- all cell expression can be edited interactively

- all changes to cells are automatically propagated through the spreadsheet

- function parameters can be bound to GUI controls for interactive exploration

- cells can contain graphs and tables containing measurements and statistics from images

Vision Spreadsheet is the product of years of development and many more years of experience working in the field of computer vision. I feel it provides an excellent environment for exploring solutions to computer vision problems. It is difficult to get a feel for an interactive environment by reading a paper. Visit http:// visionspreadsheet.com to download the application for free or to watch videos of Vision Spreadsheet in action.

\section{Overview of Vision Spreadsheet}

Figure 1 shows a screen shot of Vision Spreadsheet. There are four main areas to the GUI: the grid of cells, the current cell's statement, the shell, and the current cell's GUI controls.

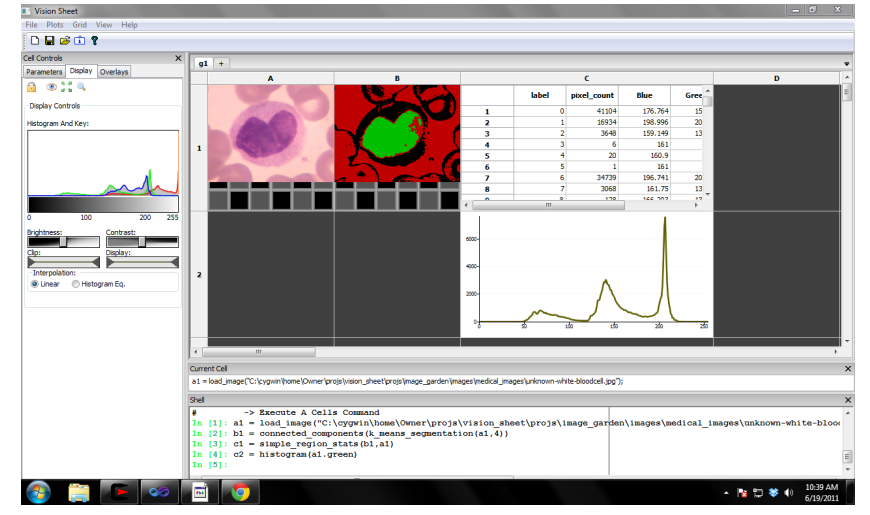

Fig. 1: Vision Spreadsheet cells contain images, measurements, and plots.

Numerical spreadsheets contain a grid of numbers and labels. Vision Spreadsheet's grid of cells contains images, computations on images, measurements, and plots. In a numerical spreadsheet, if a cell contains the sum of a column of numbers and a number in the column changes then the total automatically updates itself. Similarly, in Vision Spreadsheet if a cell changes (for instance by loading a new image or changing an algorithm parameter) then all of the cells that depend on the changed cell will update themselves. 
The current cell's statement is a single-line control used to show what statement was used to create the current cell. This statement may be edited and updated in this control.

The shell is a modified IPython shell used to specify what a cells contains. The shell is also used to write new spreadsheet functions in python.

The GUI controls area contains display parameters, overlays, and controls bound to algorithm parameters for the current cell.

\section{Specifying a Cell's Content}

Just like in a numerical spreadsheet, the content of each cell in the spreadsheet grid is defined by an expression. Expressions are entered by typing them into the shell or current cell's statement control. The syntax of Vision Spreadsheet's cell expressions should feel familiar to any spreadsheet user. But unlike a numerical spreadsheet, Vision Spreadsheet's expressions operates on images. A typical statement looks like this:

some_cell=some_function(parameter 1 , parameter 2 )

For example, to define cell b1 as the erosion of the image in cell a1 you would enter the following expression into the shell:

$\mathrm{b} 1=\operatorname{erode}(\mathrm{a} 1)$

After entering this expression, cell b1 will display the image which is an erosion of the image in cell a1. If you manually load a new image into cell a1, then the image in cell b1 will automatically update as the erosion of the new image.

The power in the expression language comes from the large library of available computer vision functions. In fact, all of the image processing and machine learning functions from OpenCV are available. This allows professionals to leverage their existing knowledge of this powerful library.

The arithmetic operators are available and follow the usual syntax and precedence rules. A typical call with an operator looks like (where someop is,,$+-<$, etc.):

some_cell = parameter1 someop parameter 2

Functions may be nested, so one way to run a morphological open would be:

$\mathrm{b} 1=\operatorname{dilate}(\operatorname{erode}(\mathrm{a} 1))$

Morphological open is already a built in functions; the above was only an example.

There are also a few special functions, like if and select.

Vision Spreadsheet supports multiple tabs per sheet. Cells in another tab are in another namespace, and can be referenced using the namespace syntax:

namespace_name: :variable_name

: :variable_name \# global namespace

Sheets start with $g$ and are sequentially numbered, so the following code is used to refer to sheet $\mathrm{g} 1$ cell a1:

g1: :a1

Literal data sets are specified with the following syntax:

$[1,2,3,4]$

$[[1,2,3],[4,5,6],[7,8,9]]$

Literal dictionaries are specified with the following syntax:

\{'name': 'Scott', 'weight':150,' location' : [512,700]\}

Keys must be a string. Values can be any supported data type (dictionaries, data sets, data frames, etc.).
Expressions can be an arbitrarily complex combination of functions and arithmetic operators. But just like in a numerical spreadsheet, cell expressions work best as simple one-line expressions. For more complex programs, use python mode within Vision Spreadsheet.

\section{Binding Parameters to GUI Controls}

A primary goal of Vision Spreadsheet is to allow interactive exploration of vision problems. One of the most powerful tools to do this is to bind GUI controls to parameters in a cell expression. This allows users to have a value in a cell expression that comes from a GUI control, such as a slider control. The user can manipulate the GUI control to affect the value in the expression. Because Vision Spreadsheet automatically propagates this change through the spreadsheet, users can very quickly see the effect that a particular parameter has on the result of an algorithm.

The best way to explain this feature is to look at an example. Consider thresholding an image. There are a couple of threshold operators, but the simplest is the ' $>$.' operator. Load an image in cell a1. Next, threshold it by typing:

$\mathrm{b} 1=\mathrm{a} 1>.128$

This creates an image where values greater than 128 are set to 255 and values less than or equal to 128 are set to zero. One way to decide on a threshold value would be to keep typing in numbers until the threshold image looked good. A better way is to bind the parameter to a GUI control, like a slider. The following command does this:

$\mathrm{b} 1=\mathrm{a} 1>$. slider $(128,0,255)$

This creates a slider with a default value of 128 , a min value of 0 , and a max value of 255. If the threshold image is the current cell, then the cell controls pane on the left of the GUI will contain a slider (see figure 2). This slider is used to interactively change the parameter to the threshold function.

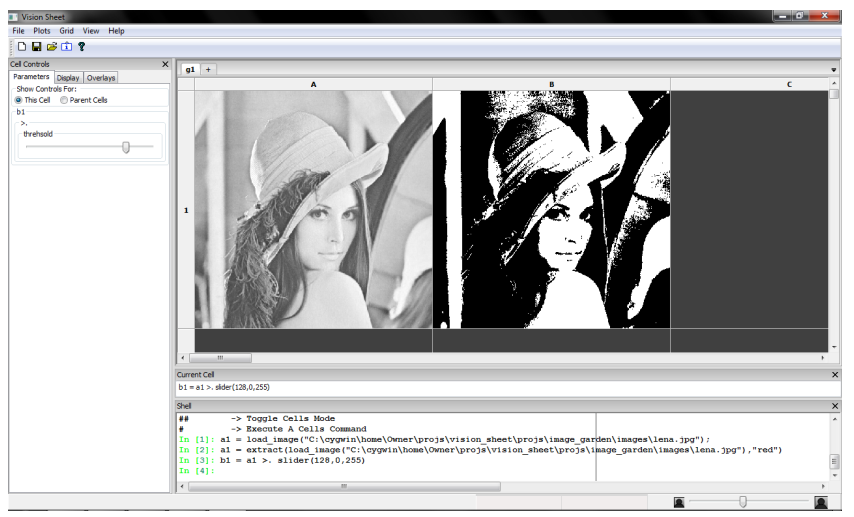

Fig. 2: GUI controls may be bound to algorithm parameters. Here a slider is bound to a threshold.

There are many other types of GUI controls that may be bound to parameters, such as: radio buttons, sliders, spin controls, combo boxes and movie controls (radio button are particularly useful to bind to file names so different images may be easily loaded into a cell).

Multiple GUI controls may be used to control a single function parameter. If the function calls to create the GUI controls are nested, then they will control the same parameter. For example, to have a spin control and a slider control the threshold:

$\mathrm{b} 1=\mathrm{a} 1>\operatorname{spin}(\operatorname{sid} \operatorname{der}(128,0,255))$ 


\section{Python Mode}

Python is a fantastic language for exploratory computer vision. Vision Spreadsheet is tightly integrated with python and gives users full access to an IPython shell within Vision Spreadsheet. This integration gives users all of the power and familiarity of python combined with the visual feedback and interactivity of Vision Spreadsheet. Users can extend Vision Spreadsheet by adding new functions they implement in python. Users also have full access to the Vision Spreadsheet environment from within python, allowing them to access and update cells interactively from within the IPython shell.

To toggle the shell to/from IPython mode, type "\#\#" in the shell. Inside the IPython shell, you will have access to the vis_sheet module. The vis_sheet module provides full access to the Vision Spreadsheet environment from within python. The IPython shell at the bottom of the GUI supports two modes, cells mode and python mode. To toggle between the two modes, type '\#\#' and hit return. Cells mode is the default mode. Python mode is just a regular IPython shell with two differences: typing '\#\#' will toggle to cells mode and there is a module called 'vis_sheet' that can be used to interact with the spreadsheet.

There are at least two interesting activities to do in python mode:

1) Extend the spreadsheet with new functions.

2) Get values from the spreadsheet, muck around with them interactively in python, and set the values back into the spreadsheet.

Here is how to add a new function to the spreadsheet. Change to python mode by typing '\#\#'. The shell should now have a black background. Define a subtraction function as follows:

def my_subtract $(a, b)$ : return $a-b$

import vis_sheet

vis_sheet.add_python_op (my_subtract)

Change back to cells mode by typing '\#\#' (the shell should now have a white background). Load an image in cell a1, erode it an put it in b1, and subtract b1 from a1 using the new function:

$\mathrm{c} 1=$ my_subtract $(\mathrm{a} 1, \mathrm{~b} 1)$

Cell c1 will contain the edges from the image in cell a1. Note that the images in the spreadsheet are automatically converted to NumPy arrays before they are passed to user defined functions. The parameters a and $b$ will be NumPy arrays. If the result is a NumPy array, it will automatically be converted to an image.

To get or set values in the spreadsheet from python mode, use the following functions:

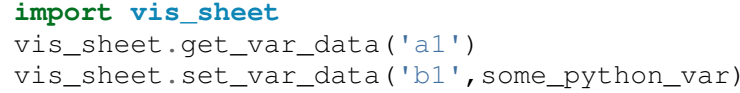

\section{Data Structures}

There are three main data structures in vision spreadsheet: images, data frames, and statistical models.

Images are the most important data structure. An image is a two dimensional array of vectors. All the elements of an image are of the same numeric type. Images with element types of uchar through double are supported. Many image types are supported, for example: grayscale, color (rgb, brg, hsi, cie lab, etc.), and depth images (from the Kinect camera, for example). When an image is passed to a user defined python function it is automatically converted into a NumPy array.
Data frames are modeled after R's data frame structure. Data frames are used to store measurements on images and to overlay images with shapes and regions of interest. It is a table where each column in the table may have a different type. So a single data frame may have a column of numbers and a column of strings. Supported column types are: numeric (uchar through double), boolean, string, and region of interest. Like R's data frames, rows may contain missing data. Data frames also support R's notion of factor columns. Factor columns are usually used to specify responses when training classifiers. Unlike R, vision spreadsheet supports grouping columns into a hierarchy. This is useful for storing higher-level objects in a data frame. For example, rectangles are stored in a data frame by grouping together four numeric columns. These rectangles may then be overlaid and edited on an image.

The last major data structure is a statistical model. Statistical models are used to classify objects in images. There are two main functions to a statistical model: train and predict. The train function takes a statistical model, a data frame of features, and a data frame of responses. It returns the newly trained model. The predict function takes a model and data frame. It returns a prediction for each row in the data frame.

There are other data types in vision spreadsheet, but many problems in computer vision can be solved using only these three data types.

\section{Conclusion}

I described a new environment for interactively working with computer vision. I am optimistic that this will be a useful and productive environment for many types of users. However, at this point no one except myself has used Vision Spreadsheet. The key to making the environment useful is to have real users try to solve real problems with it. My goal in presenting this paper is to get people using the spreadsheet so they can provide the feedback I need to make Vision Spreadsheet as useful as I know it can be. Please try it out.

I had planned on releasing Vision Spreadsheet shortly before the conference. I did not make this deadline, but I am very close. When it is released, you can go to http://visionspreadsheet.com to download it for free.

\section{Thank You}

I owe thanks to many great open source projects. I especially want to thank the following projects (alphabetical order): ANTLR $^{1}$, boost $^{2}$, CMake $^{3}$, IPython ${ }^{4}$ [IPy], OpenKinect ${ }^{5}$, $\mathrm{NumPy}^{6}, \mathrm{OpenCV}^{7}$, python $^{8}, \mathrm{SciPy}^{9}, \mathrm{SWIG}^{10}, \mathrm{VIGRA}^{11}$, 
wxPython ${ }^{12}$, and wxWidgets ${ }^{13}$.

\section{REFERENCES}

[IPy] Fernando Perez, Brian E. Granger, "IPython: A System for Interactive Scientific Computing," Computing in Science and Engineering, vol. 9, no. 3, pp. 21-29, May/June 2007, doi:10.1109/MCSE.2007.53.

1. http://www.antlr.org ANTLR is used to build the parser for the cells language.

2. http://www.boost.org. Boost is used for many utility routines in the c++ code.

3. http://www.cmake.org. CMake is the build/test/package system.

4. http://ipython.org IPython is the shell.

5. http://openkinect.org OpenKinect is the interface to the Kinect camera.

6. http://numpy.scipy.org NumPy arrays are used to interface between the internal data structures in vision spreadsheet and python.

7. http://opencv.willowgarage.com OpenCV provides many of the image processing and machine learning algorithms.

8. http://python.org. The spreadsheet is extended through python.

9. http://www.scipy.org SciPy makes it easy and efficient for the user to extend the spreadsheet and manipulate images and data frames.

10. http://www.swig.org SWIG is used to wrap OpenCV functions into Vision Spreadsheet.

11. http://hci.iwr.uni-heidelberg.de/vigra VIGRA provides many image processing algorithms.

12. http://www.wxpython.org. wxPython is the python interface to the wxwidgets library.

13. http://wxwidgets.org. wxWidgets is the GUI library. 